\title{
Strong shear-flow modulation of instabilities in rapid directional solidification
}

\author{
Katarzyna N. Kowal ${ }^{1,2,3 *}$ and Stephen H. Davis ${ }^{1}$ \\ ${ }^{1}$ Department of Engineering Sciences and Applied Mathematics, Northwestern University, 2145 Sheridan \\ Road, Evanston, IL 60208, United States \\ ${ }^{2}$ Department of Applied Mathematics and Theoretical Physics, University of Cambridge, Wilberforce Road, \\ Cambridge, CB3 0WA, United Kingdom \\ ${ }^{3}$ Trinity College, University of Cambridge, Cambridge, CB2 1TQ, United Kingdom
}

\begin{abstract}
We examine the effect of a strong shear flow on morphological instabilities that occur in the directional solidification of a dilute, binary alloy when the interface departs from local thermal equilibrium in a frozen-temperature, one-sided model. In particular, the flow velocity $U_{\infty}$ is much larger than the rate of solidification $V$ and the Schmidt number is arbitrary. In contrast to solidification processes under small or no flow, for which both a cellular and an oscillatory mode of instability appear, the liquid-solid interface under flows of large magnitude is susceptible to a single mode of instability. All experiments on banding occur in the overlap region between these modes under no flow and since these two primary modes coalesce into one time-dependent, spatially-dependent mode, there is no mechanism for the production of bands anymore in the large-flow regime. No experiments have been conducted to date on this rapid solidification under flow and this suggests that such experiments would show no bands. The flow significantly stabilizes and selects higher wavenumbers for the preferred form of instability in comparison to systems involving small or no flow. The introduction of a strong flow provides an effective mechanism to eliminate instabilities at high solidification rates. However, stability thresholds at low solidification rates remain mainly indifferent to the presence of flow.
\end{abstract}

Keywords. Additive manufacturing, binary alloys, interface, phase transition, rapid solidification. 


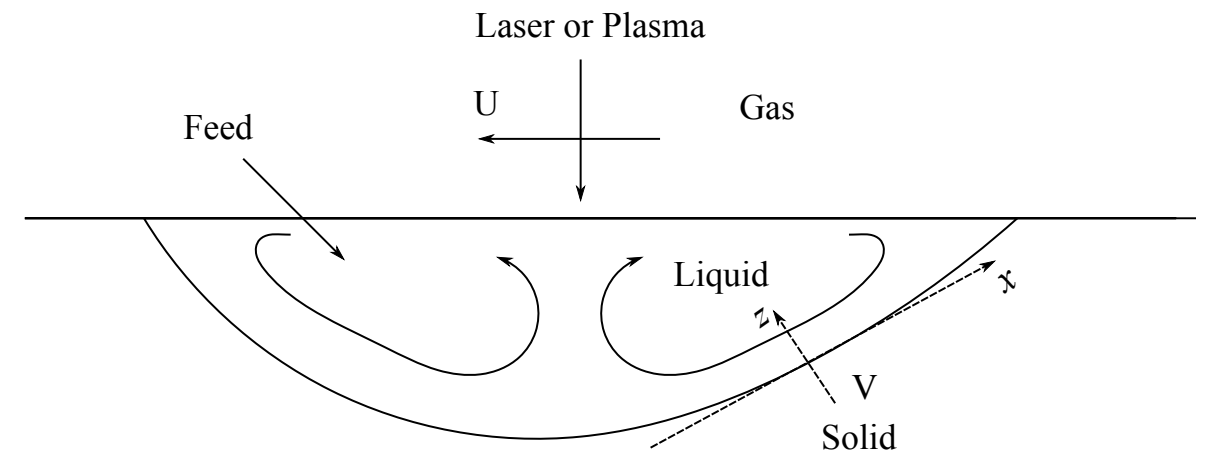

Figure 1: Liquid melt pool solidifying in additive manufacturing. Reproduced from [16].

\section{Introduction}

The production capabilities of additive manufacturing (AM), or three-dimensional printing (3DP), offer promising ways to print complex parts of diverse geometries and materials, both organic and non-organic $[22,28,12$, 9, 29, 27, 24, 21, 5, 10]. Examples range from metallic parts [28] to electronics [29] and implants and prostheses $[10]$.

However, a number of undesirable effects hinder current production capabilities of AM. These effects depend on various factors, including the temperature distribution within the melt pool, compositional changes, the geometry of the melt pool and other thermo-physical effects. Examples of these effects include distortion, compositional changes, fusion defects [22], high surface roughness, layer delamination and warping [25], and denudation [19].

To understand these effects and to minimize their occurances, it is necessary to examine the effects of the flow field within the melt pool, depicted in Fig. 1, which comes about owing to large thermal gradients, and thus large gradients in the surface tension, near the laser heat source, giving rise to Marangoni convection within the melt pool $[25,14]$. The melt pool is being scanned along the sample surface at high speed $U$, melting ahead of the heat source and solidifying rapidly behind it. The solidifying interface is near planar as depicted by the inclined line in Fig. 1. The upper, liquid-gas interface is susceptible to a number of instabilities as described by [17]. Far away from the laser heat source, the liquid solidifies rapidly and the solid-liquid interface is susceptible to morphological instabilities, which determine the microstructures of the resulting solid, such as the cellular structure typical of additive manufacturing and banding seen in analogue experiments.

Kowal et al. [16] examine the effect of a weak shear flow on these morphological instabilities, building upon the work of Merchant \& Davis [20], who examine the morphological stability of the solid-liquid interface under no flow. Both of these studies allow the solid-liquid interface to depart from thermodynamic equilibrium,

\footnotetext{
*Email address for correspondence: katarzyna.kowal@northwestern.edu
} 


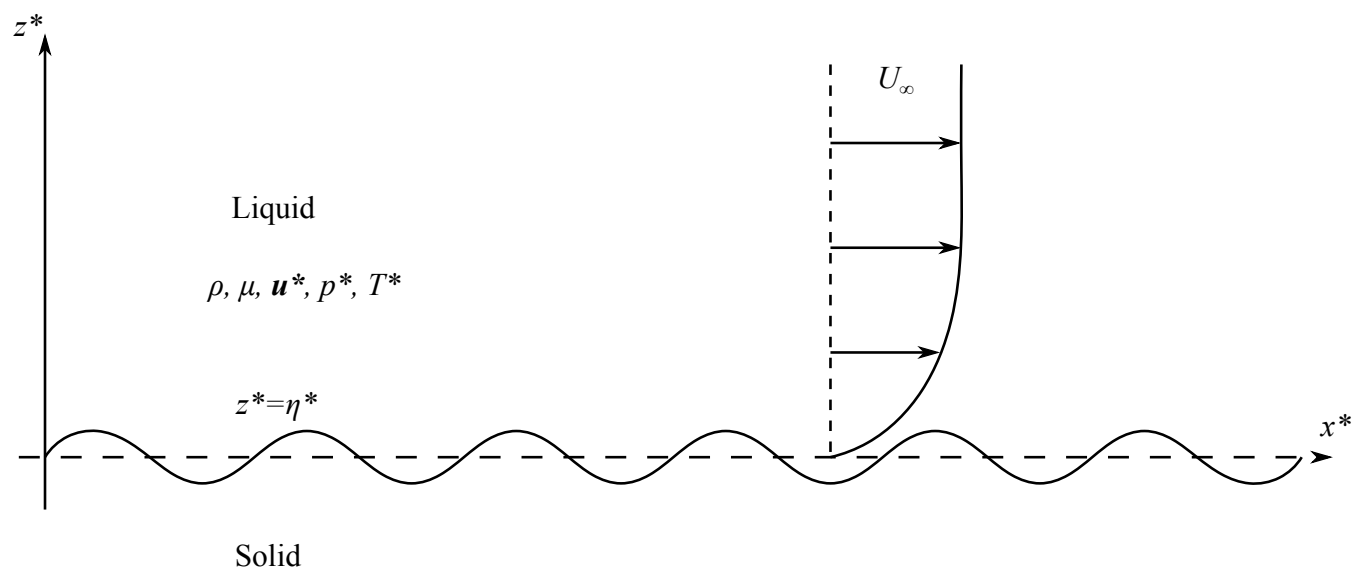

Figure 2: Schematic of the solid-liquid interface under a shear flow.

appropriate to the case at large solidification rates, which occur in AM environments. In such settings, phase transitions are no longer governed by the phase diagram. Departures from equilibrium, including effects such as solute trapping and kinetic undercooling, are formulated within thermodynamically consistent generalisations by $[2,13,1,4,3,18]$.

Merchant \& Davis [20] use this model to map out a state diagram for the different modes of instability. Two modes of instability are present when the interface departs from thermodynamic equilibrium under no flow: a steady, cellular mode and a oscillatory mode. Merchant \& Davis [20] found that all existing experimental observations of bands occur in the triangular overlap region between the two neutral curves for the two primary modes of instability.

A weak flow modulates the two modes of instability when the interface departs from thermodynamic equilibrium and travelling waves appear as shown by [16]. When the interface is kept at thermodynamic equilibrium, it has been found that an imposed shear flow also favours traveling waves [11]. Forth \& Wheeler [8] have also investigated the effect of a shear flow in the limit of large Schmidt number and large Reynolds number. The first study of morphological instabilities in the directional solidification of a binary alloy in thermodynamic equilibrium without flow has been conducted by Mullins \& Sekerka [23] and many generalizations have been provided by Coriell \& McFadden [6].

The large thermal gradients in AM environments lead to vigorous flows within the melt pool of magnitude often much larger than the solidification rate. We build upon [16], on morphological instabilities under weak flow in a rapid solidification environment, and extend the analysis to large flow rates. Specifically, we investigate the stability of the liquid-solid interface in rapid directional solidification under the influence of a boundarylayer flow, depicted in Fig. 2, of magnitude much larger than the solidification rate and develop an asymptotic analysis in this limit. We assume that the effect of flow within the melt pool affects solidification processes 
locally at the solid-liquid interface, where the flow is locally of the boundary-layer type illustrated in Fig. 2. The assumption is valid for perturbations of small amplitude characteristic of the onset of instability, which is the case in the linear theory of this paper, but may break down at sufficiently large amplitudes. In contrast to the microstructures produced in this locally-planar setting, the corresponding global effect of the solidification of the whole melt pool subject to vigorous Marangoni convection is more complex, resulting in ring-like deformations behind the flow's stagnation point. In contrast to solidification under weak flow, or no flow, once the magnitude of the flow is large, the two modes coalesce into a single mode and the region of instability is significantly reduced. Once the two modes have coalesced, there is no impetus for the generation of bands.

We begin with a theoretical development of the problem and setting out the linear stability theory in $\S 2$. We perform an asymptotic analysis to several orders in $\S 3$ and follow with a discussion of the results in $\S 4$. We revert to natural scalings, which make it possible to specify a measure of the solute concentration and solidification speed as externally controllable parameters, in $\S 5$. Implications of the results under these scalings is of use in many physical and experimental settings. We finalize with conclusions in $\S 6$.

\section{Theoretical development}

The geometry of the problem is set up in the schematic of Fig. 2. A dilute, binary alloy is directionally solidified in the $z^{*}$-direction and the coordinate system is chosen so that the unperturbed solid-liquid interface is at $z^{*}=0$. Liquid occupies the region $z^{*}>\eta^{*}$, and solid occupies the region $z^{*}<\eta^{*}$, where $z=\eta^{*}$ is the position of the perturbed interface. A strong shear flow is imposed orthogonal to the direction of solidification, with far-field velocity $U_{\infty}$. These and other symbols are defined in Table 1.

The fluid is assumed incompressible, that there is no change in the density of the material as it changes phase, and that the effect of gravity is negligible. The solid-liquid interface is allowed to depart from thermodynamic equilibrium and make the following assumptions for the thermal and solutal problems. Assume that $(a)$ the thermal diffusivities of the solid and liquid phases are much larger than the diffusivity of solute in the liquid phase, (b) the diffusivity of the solute is negligible in the solid phase, $(c)$ the thermal conductivities of both phases are equal, and $(d)$ latent heat production at the interface can be neglected, These are the assumptions adopted by [20] and [16]. Assumptions $(c)$ and $(d)$ allow the thermal field to fully decouple from the solutal and flow fields, rendering the analysis more tractable and the interpretation of the results clearer.

In rapid solidification environments, phase transitions are no longer governed by the equilibrium phase diagram. [2, 4, 3, 18] developed a thermodynamically consistent model for phase transitions that depart from interfacial equilibrium, which specifies a non-equilibrium local interfacial temperature $T_{I}^{*}$ and local solute 


\begin{tabular}{ll} 
Symbol & Definition \\
\hline$\eta^{*}$ & Interface deflection \\
$U_{\infty}$ & Far-field velocity \\
$T_{M}$ & Equilibrium melting temperature of the pure material \\
$m_{E}$ & Equilibrium liquidus slope \\
$k_{E}$ & Equilibrium segregation coefficient \\
$\gamma / L_{\nu}$ & Capillarity constant \\
$V_{0}$ & Upper bound for the rate at which crystallization can occur \\
$\mathcal{M}$ & Morphological number \\
$\beta$ & Disequilibrium parameter \\
$\mathcal{U}$ & Attachment-kinetics parameter \\
$k_{E}$ & Segregation coefficient \\
$\Gamma$ & Dimensionless surface energy \\
$\mathcal{V}$ & Speed ratio \\
$\mathcal{R}$ & Inverse Schmidt number \\
\hline
\end{tabular}

Table 1: Table of parameters

concentration $C_{s}^{*}$ in the solid.

The former is given by

$$
T_{I}^{*}=T_{M}\left(1+2 H^{*} \frac{\gamma}{L_{\nu}}\right)+m^{*}\left(V_{n}^{*}\right) C^{*}-\frac{m_{E}}{k_{E}-1} \frac{V_{n}^{*}}{V_{0}},
$$

which incorporates the Gibbs-Thomson effect for curved interfaces and the effects of capillary undercooling and kinetic undercooling. This relationship appears e.g. in [20] and [7], and was originally derived by following the arguments of [4] and [3] for a planar interface.

The constants appearing in this relation are described as follows. $T_{M}$ is the equilibrium melting temperature of the pure material, $m_{E}$ is the equilibrium liquidus slope, $k_{E}$ is the equilibrium segregation coefficient, $\gamma / L_{\nu}$ is the capillarity constant, and $V_{0}$ is the upper bound for the rate at which crystallization can occur.

The liquidus slope due to non-equilibrium segregation is no longer a constant, as in interfacial equilibrium, but a function of the local interface velocity $V_{n}^{*}$. Its functional form is given by

$$
m^{*}\left(V_{n}^{*}\right)=m_{E}\left\{1-\frac{1}{k_{E}-1}\left(k_{E}-k^{*}\left(V_{n}\right)\left[1-\ln \left(\frac{k^{*}\left(V_{n}^{*}\right)}{k_{E}}\right)\right]\right)\right\} .
$$

The remaining quantities appearing in (1) are the mean curvature $H^{*}$, which for a non-planar, three- 
dimensional surface becomes

$$
2 H^{*}=\frac{\eta_{x^{*} x^{*}}^{*}\left(1+\eta_{y^{*}}^{* 2}\right)-2 \eta_{x^{*}}^{*} \eta_{y^{*}}^{*} \eta_{x^{*} y^{*}}^{*}+\eta_{y^{*} y^{*}}^{*}\left(1+\eta_{x^{*}}^{* 2}\right)}{\left(1+\eta_{y^{*}}^{* 2}+\eta_{x^{*}}^{* 2}\right)^{3 / 2}},
$$

and the solidification rate for non-planar growth

$$
V_{n}^{*}=\frac{V+\eta_{t^{*}}^{*}}{\left(1+\left|\nabla^{*} \eta^{*}\right|^{2}\right)^{1 / 2}}
$$

The latter ingredient in the non-equilibrium model, namely $C_{s}^{*}$, is related to the local solute concentration in the liquid via the relationship

$$
C_{s}^{*}=C^{*} k^{*}\left(V_{n}^{*}\right)
$$

The segregation coefficient $k^{*}$ is no longer fixed, as in interfacial equilibrium, but a function of the local interface velocity $V_{n}^{*}$ given by

$$
k^{*}\left(V_{n}^{*}\right)=\frac{k_{E}+\beta_{0} V_{n}^{*}}{1+\beta_{0} V_{n}^{*}} .
$$

[13] and [1] determined this particular functional form consistently with observation and limiting values so that at low solidification rates, the segregation coefficient is close to its equilibrium value $k_{E}$, and at high solidification rates, the segregation coefficient $k^{*} \rightarrow 1$, reflecting the observation that solute is trapped into the solid at these rates.

\subsection{Dimensionless equations}

The governing equations are made dimensionless by scaling the velocity field by the far-field speed, scaling time and space based on solute diffusion, $t^{*} \sim \mathcal{D} / U_{\infty}^{2}$ and $l^{*} \sim \mathcal{D} / U_{\infty}$, and adopting the viscous pressure scaling

$$
p^{*}=\frac{\mu U_{\infty}^{2}}{\mathcal{D}} p
$$

The thermal and solutal fields are scaled as

$$
T=\frac{T^{*}-T_{0}}{G \mathcal{D} / U_{\infty}}, \quad C=\frac{C^{*}-C_{\infty} / k_{E}}{C_{\infty}\left(k_{E}-1\right) / k_{E}}
$$

Here, $G$ is the imposed thermal gradient and $T_{0}$ is a reference equilibrium freezing temperature of the material.

In dimensionless form, the governing equations read

$$
\nabla^{2} T=0
$$

describing heat balance,

$$
\frac{\partial C}{\partial t}-\mathcal{V}^{-1} \frac{\partial C}{\partial z}+\boldsymbol{u} \cdot \nabla C=\nabla^{2} C
$$


describing solute balance,

$$
\mathcal{R}\left(\frac{\partial \boldsymbol{u}}{\partial t}-\mathcal{V}^{-1} \frac{\partial \boldsymbol{u}}{\partial z}+\boldsymbol{u} \cdot \nabla \boldsymbol{u}\right)=-\nabla p+\nabla^{2} \boldsymbol{u}
$$

describing momentum balance, and

$$
\nabla \cdot \boldsymbol{u}=\mathbf{0}
$$

describing incompressibility, for the liquid $(z<\eta)$ and

$$
\nabla^{2} T=0
$$

describing heat balance for the solid $(z<\eta)$. The interfacial conditions read

$$
\begin{gathered}
C_{s}=\frac{1}{1-k_{E}}+\left(C-\frac{1}{1-k_{E}}\right) k\left(V_{n}\right) \\
\left(C-C_{s}\right) V_{n}=-\left(-C_{x} \eta_{x}-C_{y} \eta_{y}+C_{z}\right)\left(1+|\nabla \eta|^{2}\right)^{-1 / 2} \\
T=2 H \mathcal{M} \Gamma+\mathcal{M}\left[C+\left(m\left(V_{n}\right)-1\right)\left(\frac{1}{k_{E}-1}+C\right)\right]-\mathcal{M U} V_{n}
\end{gathered}
$$

at $z=\eta$ and the far field conditions become

$$
\begin{gathered}
u \rightarrow \boldsymbol{e}_{\boldsymbol{x}} \quad \text { as } \quad z \rightarrow \infty \\
C \rightarrow 1 \quad \text { as } \quad z \rightarrow \infty
\end{gathered}
$$

The segregation coefficient and the liquidus slope are now dimensionless functions of the solidification rate,

$$
\begin{gathered}
k\left(V_{n}\right)=\frac{k_{E}+\beta V_{n}}{1+\beta V_{n}}, \\
m\left(V_{n}\right)=1+\frac{1}{1-k_{E}}\left[k_{E}-k\left(V_{n}\right)\left(1-\ln \frac{k\left(V_{n}\right)}{k_{E}}\right)\right],
\end{gathered}
$$

where the normal velocity is given by

$$
V_{n}=\frac{\mathcal{V}^{-1}+\eta_{t}}{\left(1+|\nabla \eta|^{2}\right)^{1 / 2}}
$$

and the mean curvature

$$
2 H=\frac{\eta_{x x}\left(1+\eta_{y}^{2}\right)-2 \eta_{x} \eta_{y} \eta_{x y}+\eta_{y y}\left(1+\eta_{x}^{2}\right)}{\left(1+\eta_{y}^{2}+\eta_{x}^{2}\right)^{3 / 2}} .
$$

These equations are governed by seven dimensionless parameters, including the morphological number $\mathcal{M}$, the disequilibrium parameter $\beta$, the attachment-kinetics parameter $\mathcal{U}$, the segregation coefficient $k_{E}$, the dimensionless surface energy $\Gamma$, the speed ratio $\mathcal{V}$, and the inverse Schmidt number $\mathcal{R}$, where

$$
\begin{gathered}
\mathcal{M}=\frac{m_{E}\left(k_{E}-1\right) C_{\infty} U_{\infty}}{\mathcal{D}_{l} G k_{E}}, \quad \beta=\beta_{0} U_{\infty}, \quad \mathcal{U}=\frac{U_{\infty} k_{E}}{\left(k_{E}-1\right)^{2} C_{\infty} V_{0}}, \\
\Gamma=\frac{T_{M} U_{\infty} k_{E}}{L_{\nu} \mathcal{D}_{l} m_{E}\left(k_{E}-1\right) C_{\infty}}, \quad \mathcal{V}=U_{\infty} / V, \quad \mathcal{R}=\frac{\rho \mathcal{D}}{\mu}
\end{gathered}
$$

These are all scaled with respect to the external flow speed. 


\subsection{Linear stability analysis}

Perturb about the basic state given by the solution

$$
\begin{aligned}
& T_{0}=z+\mathcal{M}\left(\frac{1}{1-k_{E}}-\frac{\left(\beta+k_{E} \mathcal{V}\right) \bar{\Gamma}_{s}}{\left(1-k_{E}\right) \mathcal{V}^{2}}-\frac{\mathcal{U}}{\mathcal{V}}\right), \\
& C_{0}=1-\frac{k_{E} \mathcal{V}}{k_{E} \mathcal{V}+\beta} e^{-z / \mathcal{V}} \\
& u_{0}=1-e^{-z \mathcal{R} / \mathcal{V}} \\
& \eta_{0}=v_{0}=w_{0}=p_{0}=0
\end{aligned}
$$

where

$$
\bar{\Gamma}_{s}=\frac{k_{E} \mathcal{V}^{2}}{\left(1-k_{E}\right)\left(\beta+k_{E} \mathcal{V}\right)^{2}}\left(\left(\beta+k_{E} \mathcal{V}\right) \ln \left(\frac{\beta+k_{E} \mathcal{V}}{k_{E}(\beta+\mathcal{V})}\right)+\left(1-k_{E}\right) \mathcal{V}\right)
$$

and expand in the small parameter $\epsilon$ by writing

$$
\begin{aligned}
X(\boldsymbol{x}, t) & =X_{0}(z)+\epsilon X_{1}(z) e^{\sigma t+i \boldsymbol{\alpha} \cdot \boldsymbol{x}}, \\
X & =(\boldsymbol{u}, p, C)^{T},
\end{aligned}
$$

where $\boldsymbol{\alpha}=\left(\alpha_{1}, \alpha_{2}\right)$ is the horizontal wavevector with amplitude $\alpha$, and

$$
\eta(x, y, t)=\eta_{1} e^{\sigma t+i \boldsymbol{\alpha} \cdot \boldsymbol{x}}
$$

A single equation is obtained for the vertical velocity, given by,

$$
\begin{aligned}
{\left[\mathcal{V}\left(\mathcal{V} \frac{d^{2}}{d z^{2}}+\mathcal{R} \frac{d}{d z}-\mathcal{V}\left(\alpha^{2}+\sigma \mathcal{R}+i \alpha_{1} \mathcal{R}\left(1-e^{-z \mathcal{R} / \mathcal{V}}\right)\right)\right)\right.} \\
\left.\cdot\left(\alpha^{2}-\frac{d^{2}}{d z^{2}}\right)+i \alpha_{1} \mathcal{R}^{3} e^{-z \mathcal{R} / \mathcal{V}}\right] w_{1}(z)=0
\end{aligned}
$$

by eliminating pressure and the horizontal velocity. The solute conservation equation reduces to

$$
\mathcal{V} C_{1}^{\prime \prime}(z)+C_{1}^{\prime}(z)-\mathcal{V} C_{1}(z)\left(\alpha^{2}+\sigma+i \alpha_{1}\left(1-e^{-z \mathcal{R} / \mathcal{V}}\right)\right)=\frac{\mathcal{V} k_{E}}{\beta+\mathcal{V} k_{E}} w_{1}(z) e^{-z / \mathcal{V}}
$$

These are subject to the interfacial conditions

$$
\begin{gathered}
w_{1}=0, \\
w_{1}^{\prime}=i \alpha_{1} \mathcal{R} \mathcal{V}^{-1} \eta_{1}, \\
(\beta+\mathcal{V}) C_{1}^{\prime}+C_{1}\left(1-k_{E}\right)=\left(\frac{\sigma \mathcal{V}^{2}}{\beta+k_{E} \mathcal{V}}+\frac{1}{\mathcal{V}}\right) k_{E} \eta_{1},
\end{gathered}
$$

and

$$
C_{1}=\left(\alpha^{2} \Gamma+\sigma\left(\mathcal{U}-\mathcal{U}_{s}\right)+\mathcal{M}^{-1}+\frac{\Gamma_{s}\left(\beta \sigma \mathcal{V}^{2}-\beta-k_{e} \mathcal{V}\right)}{\mathcal{V}^{2}(\beta+\mathcal{V})}\right) \frac{\mathcal{U}_{s}(\beta+\mathcal{V})^{2}}{\beta \mathcal{V} \Gamma_{s}} \eta_{1}
$$


at $z=0$, and the far field conditions

$$
w_{1}, w_{1}^{\prime}, C_{1} \rightarrow \infty, \quad \text { as } \quad z \rightarrow \infty
$$

where

$$
\mathcal{U}_{s}=\frac{\beta k_{E} \mathcal{V}^{3}}{(\beta+\mathcal{V})\left(\beta+k_{E} \mathcal{V}\right)^{2}}
$$

The relations above form a sixth order system of differential equations subject to four interfacial conditions and three decay conditions. This makes the problem fully specified. The system is a differential eigenvalue problem for $\left(w_{1}(z), C_{1}(z), \eta_{1}\right)$, where the growth rate $\sigma$ is treated as the eigenvalue. As such, the solutions are specified only up to a constant multiple, which we fix, without loss of generality, by setting $\eta_{1}=1$.

\section{Asymptotics for $\mathcal{V} \gg 1$}

The external-flow ratio is taken to be large using an asymptotic expansion in $\mathcal{V}^{-1}$ as follows:

$$
\begin{aligned}
& w_{1}=w_{10}+\mathcal{V}^{-1} w_{11}+\mathcal{V}^{-2} w_{12}+\cdots, \\
& C_{1}=C_{10}+\mathcal{V}^{-1} C_{11}+\mathcal{V}^{-2} C_{12}+\cdots
\end{aligned}
$$

The neutral stability boundary has $\sigma=i \omega$, where $\omega \in \mathbb{R}$ is the angular frequency of oscillations. It is appropriate to expand

$$
\omega=\omega_{0}+\mathcal{V}^{-1} \omega_{1}+\mathcal{V}^{-2} \omega_{2}+\cdots
$$

The neutral stability curve is determined by the inverse morphological number, which we expand as

$$
\mathcal{M}^{-1}=\mathcal{V}^{-1} m_{0}+\mathcal{V}^{-2} m_{1}+\cdots
$$

\subsection{Zeroth-order problem}

At zeroth order in $\mathcal{V}^{-1}$, the governing equations reduce to

$$
\begin{aligned}
& w_{10}^{(4)}(z)+w_{10}^{\prime \prime}(z)\left(2 \alpha^{2}+i \omega_{0} \mathcal{R}\right)+w_{10}(z)\left(\alpha^{4}+i \alpha^{2} \omega_{0} \mathcal{R}\right)=0 \\
& C_{10}^{\prime \prime}(z)-\left(\alpha^{2}+i \omega_{0}\right) C_{10}(z)=0
\end{aligned}
$$

subject to the interfacial conditions

$$
\begin{gathered}
w_{10}=0, \quad w_{10}^{\prime}=0, \quad C_{10}^{\prime}-i \omega_{0}=0, \\
C_{10}=\alpha^{2} \Gamma+m_{0}+i \mathcal{U} \omega_{0},
\end{gathered}
$$


at $z=0$, and the far field conditions

$$
w_{10}, w_{10}^{\prime}, C_{10} \rightarrow 0, \quad \text { as } \quad z \rightarrow \infty
$$

The solution is given by

$$
w_{10}(z)=0, \quad C_{10}(z)=0,
$$

and the solvability condition gives

$$
m_{0}=-\alpha^{2} \Gamma-i \omega_{0}\left(\mathcal{U}+\frac{1}{\sqrt{\alpha^{2}+i \omega_{0}}}\right)
$$

Requiring $m_{0}$ to be real gives an equation for the zeroth-order frequency

$$
\omega_{0}\left[\mathcal{U}+\left(\alpha^{4}+\omega_{0}^{2}\right)^{-1 / 4} \cos \left(\frac{1}{2} \arg \left(\alpha^{2}+i \omega_{0}\right)\right)\right]=0
$$

from which we deduce the solution $\omega_{0}=0$ and

$$
m_{0}=-\alpha^{2} \Gamma
$$

At leading order, the system only feels the effects of surface energy and is strongly stabilized by it for all wavenumbers.

\subsection{First-order problem}

At first order in $\mathcal{V}^{-1}$, the governing equations become

$$
\begin{aligned}
& w_{11}^{(4)}(z)-2 \alpha^{2} w_{11}^{\prime \prime}(z)+\alpha^{4} w_{11}(z)=0 \\
& C_{11}^{\prime \prime}(z)-\alpha^{2} C_{11}(z)=0
\end{aligned}
$$

subject to

$$
\begin{gathered}
w_{11}=0, \quad w_{11}^{\prime}-i \alpha_{1} \mathcal{R}=0, \\
C_{11}^{\prime}=i \omega_{1}, \\
C_{11}=i \mathcal{U} \omega_{1}+m_{1}-1,
\end{gathered}
$$

at $z=0$, and

$$
w_{11}, w_{11}^{\prime}, C_{11} \rightarrow 0 \quad \text { as } \quad z \rightarrow \infty .
$$

Note that there is no viscous boundary layer. The solution is given by

$$
w_{11}=i \alpha_{1} \mathcal{R} z e^{-\alpha z}, \quad C_{11}=-i \alpha^{-1} \omega_{1} e^{-\alpha z},
$$


and the solvability condition at this order becomes

$$
\alpha\left(1-m_{1}\right)=i \omega_{1}(\alpha \mathcal{U}+1) .
$$

Requiring for the morphological number to be real specifies the first-order frequency

$$
\omega_{1}=0
$$

and the first-order correction to the inverse morphological number

$$
m_{1}=1 .
$$

The positivity of this higher-order term implies that the strong shear flow does not stabilize the system completely. There is an interval of small wavenumbers that give rise to instability.

\subsection{Second-order problem}

At second order in $\mathcal{V}^{-1}$, the governing equations reduce to

$$
\begin{aligned}
w_{12}{ }^{(4)}(z)-2 \alpha^{2} w_{12}^{\prime \prime}(z)+\alpha^{4} w_{12}(z)= & -\mathcal{R} w_{11}{ }^{(3)}(z)+i \alpha_{1} \mathcal{R}^{2} z w_{11}^{\prime \prime}(z) \\
& +\alpha^{2} \mathcal{R} w_{11}^{\prime}(z)-i \alpha_{1} \alpha^{2} \mathcal{R}^{2} z w_{11}(z), \\
\alpha^{2} C_{12}(z)-C_{12}^{\prime \prime}(z)= & -w_{11}(z)+C_{11}^{\prime}(z)-i \alpha_{1} z \mathcal{R} C_{11}(z),
\end{aligned}
$$

subject to

$$
\begin{gathered}
w_{12}=0, \quad w_{12}^{\prime}=0 \\
C_{12}^{\prime}+\left(1-k_{E}\right)\left(C_{11}-\beta C_{10}\right)=k_{E}+i \omega_{2}, \\
C_{12}=\frac{\beta^{2}\left(k_{E}-1\right)}{2 k_{E}} C_{10}+\frac{\beta}{k_{E}}+m_{2}+i \mathcal{U} \omega_{2},
\end{gathered}
$$

at $z=0$ and

$$
w_{12}, w_{12}^{\prime}, C_{12} \rightarrow 0 \quad \text { as } \quad z \rightarrow \infty .
$$

The solution is given by

$$
\begin{aligned}
w_{12} & =\left[\frac{1}{12} \alpha_{1}^{2} \alpha^{-1} \mathcal{R}^{3} z^{3}+\frac{1}{4}\left(\alpha_{1}^{2} \alpha^{-2} \mathcal{R}^{3}-i \alpha_{1} \mathcal{R}^{2}\right) z^{2}\right] e^{-\alpha z} \\
C_{12} & =-\frac{1}{4 \alpha^{3}}\left[i \alpha_{1} \alpha^{2} z^{2} \mathcal{R}+i \alpha_{1} \alpha z \mathcal{R}+4 i \alpha^{2} \omega_{2}+4 \alpha^{2} k_{E}+i \alpha_{1} \mathcal{R}\right] e^{-\alpha z},
\end{aligned}
$$

and the solvability condition at this order reduces to

$$
m_{2}=-\frac{k_{E}}{\alpha}-\frac{\beta}{k_{E}}-\frac{i \omega_{2}(\alpha \mathcal{U}+1)}{\alpha}-\frac{i \alpha_{1} \mathcal{R}}{4 \alpha^{3}} .
$$




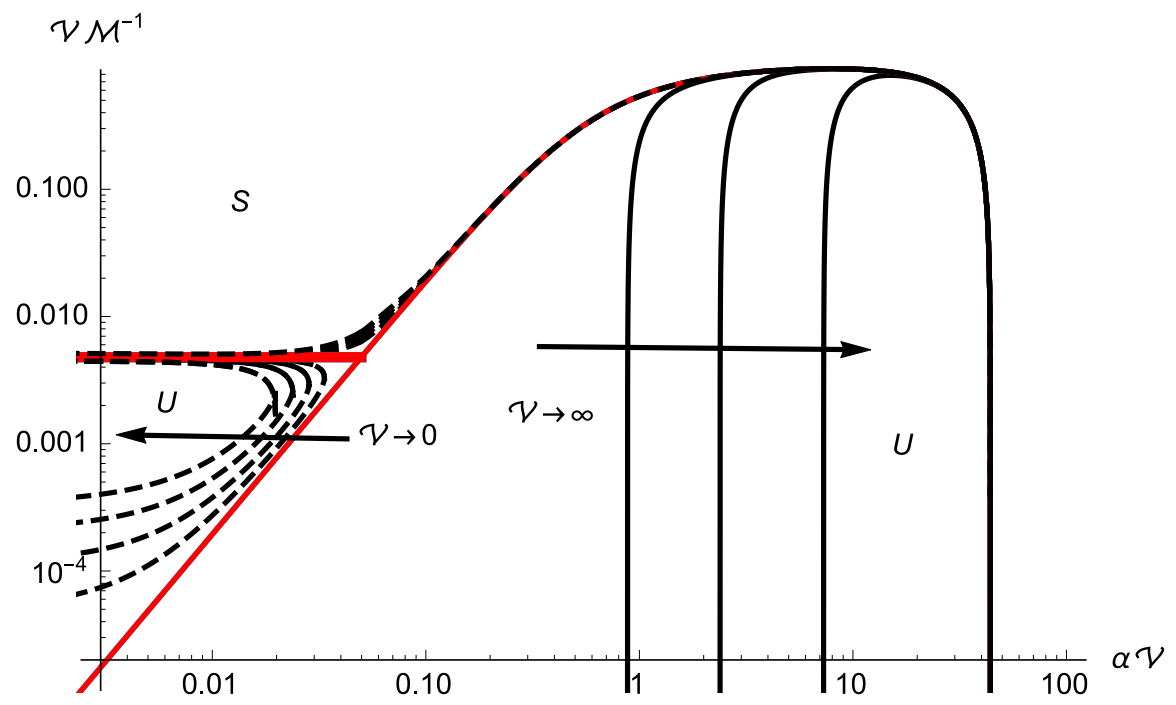

Figure 3: Neutral curves as a function of the wavenumber for $k_{E}=0.5, \beta / \mathcal{V}=0.01, \Gamma / \mathcal{V}=0.0005$. The axes are scaled based on scalings involving the solidification rate rather than the external flow velocity, as appropriate for comparison with results for weak or no flow. Neutral curves for large $\mathcal{V}$ are shown as black, solid curves for $\mathcal{V}=1,10,100$. Neutral curves for small $\mathcal{V}$ are shown as dashed curves for $\mathcal{V}=0.1,0.15,0.2,0.25$ (see [16]). For comparison, the neutral curves for $\mathcal{V}=0$ are shown as solid, red curves. The unstable region is the region underneath the solid curves.

Requiring $m_{2}$ to be real yields the first nonzero term in the asymptotic expansion for the frequency. That is,

$$
\omega_{2}=-\frac{\alpha_{1} \mathcal{R}}{4 \alpha^{2}(\alpha \mathcal{U}+1)}
$$

The corresponding correction to the inverse morphological number reads

$$
m_{2}=-\frac{k_{E}}{\alpha}-\frac{\beta}{k_{E}}
$$

The effects of disequilibrium are first seen at this order and are stabilizing.

The asymptotic results, at the first three orders, also imply that there is just one instability mode for large $\mathcal{V}$, in contrast to the same scenario for small $\mathcal{V}$ or $\mathcal{V}=0$, and that this mode is oscillatory.

\section{Discussion}

Without any flow, there are two modes of instability: a steady, cellular mode and a time-periodic, oscillatory mode [20]. Once a weak $(\mathcal{V} \ll 1)$ shear flow orthogonal to the direction of the advancing solidification front is imposed, travelling waves due to the flow appear as shown by [16]. That is, both modes become oscillatory. However, these modes are still distinguishable for $\mathcal{V} \ll 1$. 


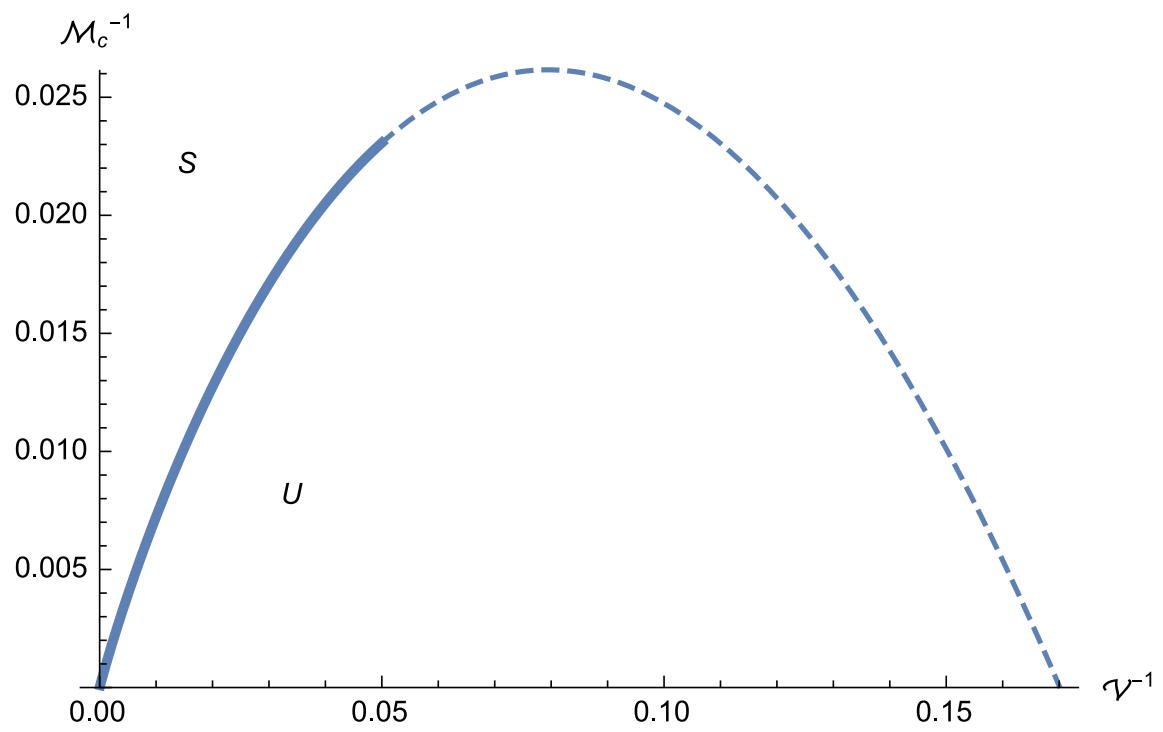

Figure 4: Critical morphological number as a function of $\mathcal{V}$ for $k_{E}=0.5, \beta=1, \Gamma=1$. The region of validity is for $\mathcal{V}^{-1} \ll 1$ as indicated by the bold, solid curve. The regions of stability are indicated.

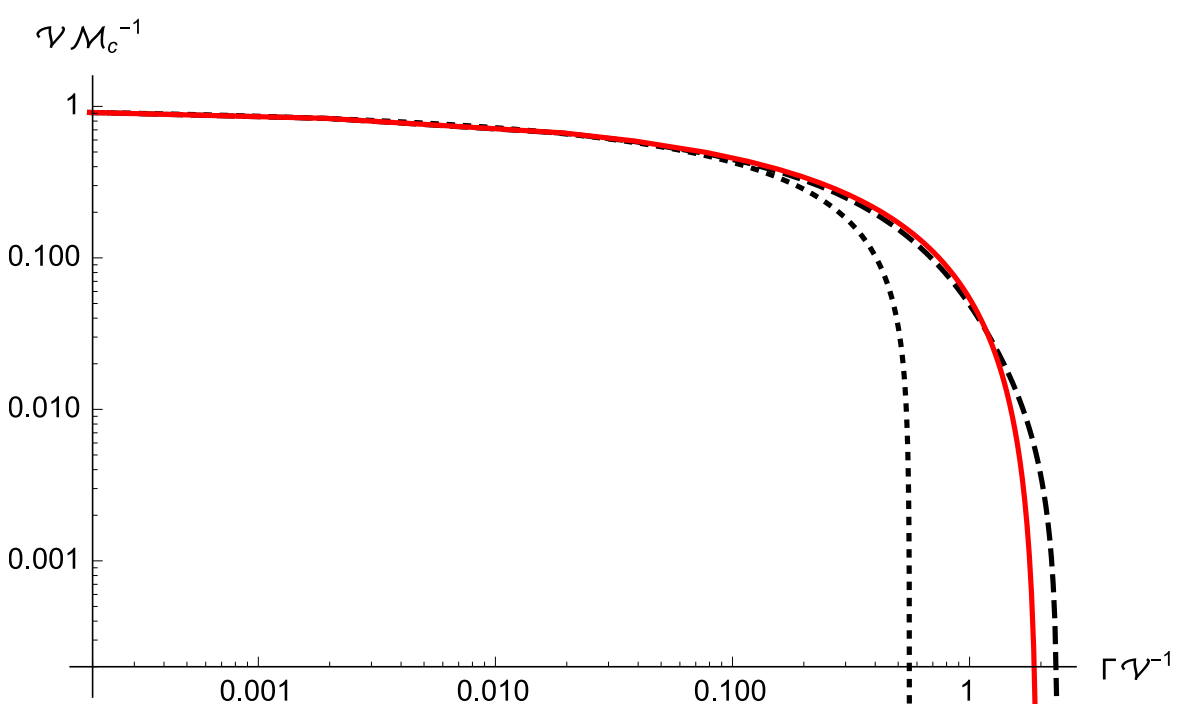

Figure 5: Critical morpholocial number for varying surface energies for large flow $(\mathcal{V}=100$ - dotted curve), weak flow $(\mathcal{V}=1$ - dashed curve, see [16]) and no flow $(\mathcal{V}=0$ - solid curve). The axes are rescaled based on scales involving the solidification rate rather than external flow rate, as appropriate for comparison with results involving weak or no flow. Parameters used: $k_{E}=0.5, \beta \mathcal{V}^{-1}=0.01$. 


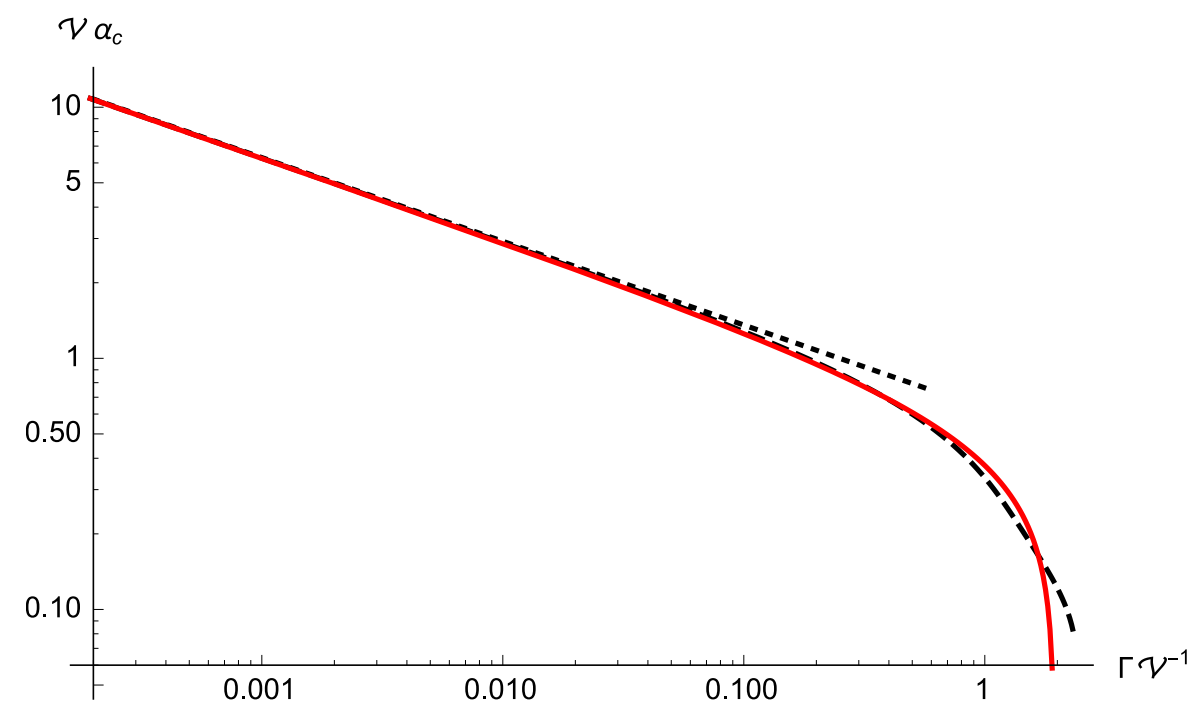

Figure 6: The critical wavenumber $\alpha_{c}$ for varying surface energies for large flow $(\mathcal{V}=100$ - dotted curve), weak flow $(\mathcal{V}=1$ - dashed curve) and no flow $(\mathcal{V}=0$ - solid curve). The axes are rescaled based on scales involving the solidification rate rather than external flow rate, as appropriate for comparison with results involving weak or no flow. Parameters used: $k_{E}=0.5, \beta \mathcal{V}^{-1}=0.01$.

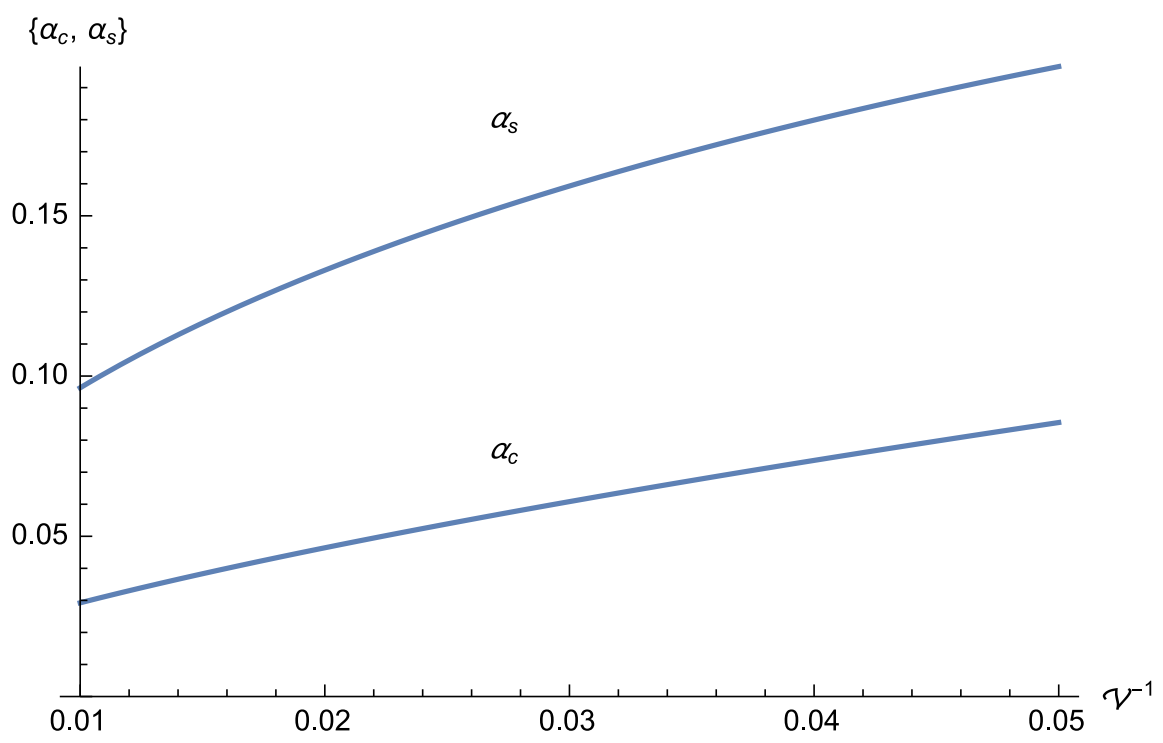

Figure 7: The critical wavenumber $\alpha_{c}$ and cutoff wavenumber $\alpha_{s}$ for varying $\mathcal{V}^{-1}$. The axes are scaled based on the external flow rate. Parameters used: $k_{E}=0.5, \beta=1, \Gamma=1$. 


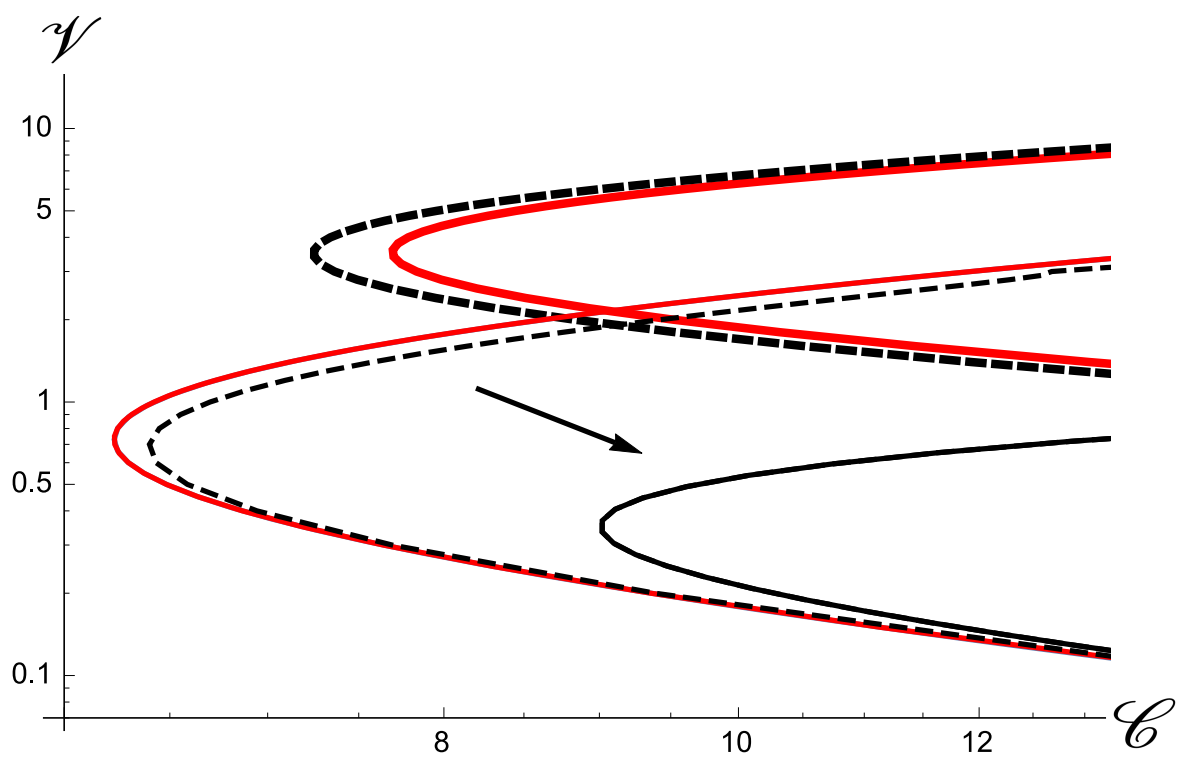

Figure 8: Neutral stability curves in rescaled variables $(\mathscr{C}, \mathscr{V})$. Leading-order results for $\mathcal{V}=10$ are shown in black. For comparison, results for no and weak flow are overlain. The steady and oscillatory branches under no flow are given by the solid thin and thick red curves. The weak-flow perturbations to these brances are shown as dashed thin and thick curves, respectively and are reproduced from [16]. Parameters used: $k_{E}=0.8, \mathscr{B}=0.3$, $\mathscr{N}=0.05$.

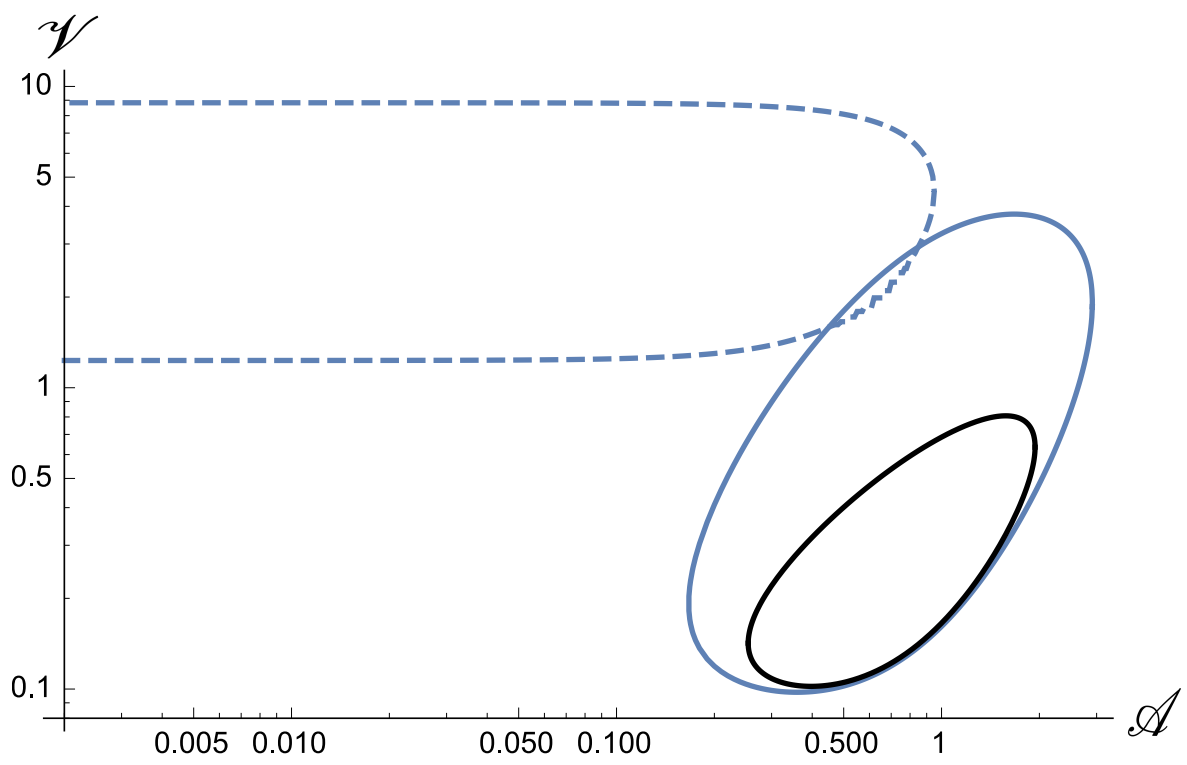

Figure 9: Neutral stability curves in rescaled variables in the $(\mathscr{A}, \mathscr{V})$ plane. This figure is a cross-section of figure 8 at $\mathscr{C}=15$. Results for $\mathcal{V}=10$ are shown in black. For comparison, results for no flow are overlain in blue. The steady and oscillatory branches under no flow are shown as solid and dashed blue curves. Parameters used: $k_{E}=0.8, \mathscr{B}=0.3, \mathscr{N}=0.05$. 
Once the magnitude of the flow is large $(\mathcal{V} \gg 1)$, these two modes become replaced by a single loop. The neutral curves for a sequence of typical parameters are shown in Fig. 3 for $\alpha_{2}=0$ in comparison to results for weak or no flow. As in [16] and [20], we focus on examining the two-dimensional setting with $\alpha_{2}=0$, for simplicity and for direct comparison to results for $\mathcal{V}=0$ and $\mathcal{V} \ll 1$. The neutral curves for $\mathcal{V} \gg 1$ are unaffected by this assumption as the expansion for the morphological number depends only on the magnitude $\alpha$, which is symmetric in $\alpha_{1}$ and $\alpha_{2}$. Symmetry is lost in the expansion for the frequency $\omega$, which is given additionally in terms of $\alpha_{1}$ and the leading order term in the expansion for $\omega$ vanishes when $\alpha_{1}=0$. If $\alpha_{1}, \alpha_{2}=0$, it is still mathematically appropriate to reduce the governing equations to one involving the vertical component of the perturbed velocity and results such as the occurance of banding are unaffected by the choice to set $\alpha_{2}=0$.

When the magnitude of the flow is small, the perturbed oscillatory mode moves to the left until it is no longer seen. The critical $\mathcal{M}_{c}^{-1}$ necessary for any instability to occur decreases with $\mathcal{V}$ as $\mathcal{V} \rightarrow \infty$, as shown in 4. The asymptotic results are valid for $\mathcal{V} \gg 1$ and the figure should only be interpreted in this range.

The critical wavenumber $\alpha_{c}$, corresponding to $\mathcal{M}_{c}^{-1}$, is small and finite, in contrast to that of the oscillatory mode under no flow, for which $\alpha_{c}=0$. That is, there is spatial structure for the preferred mode when $\mathcal{V} \gg 1$, in contrast to the preferred oscillatory mode under no flow, which is spatially homogenous.

The critical $\mathcal{M}_{c}^{-1}$ also decreases with $\Gamma$ until an absolute stability limit is reached at $\Gamma=\Gamma_{s}$, as shown in Fig. 5. No instabilities occur for $\Gamma>\Gamma_{s}$. A similar absolute stability limit exists for no flow. However, under strong flow, the critical wavenumber $\alpha_{c}$ is nonzero at the absolute stability limit as shown in Fig. $6\left(\alpha_{c}=0\right.$ at the absolute stability limit when no flow is present). That is, long waves are preferred near the absolute stability limit under no flow, which is no longer the case for $\mathcal{V} \gg 1$. Fig. 6 also shows a comparison to results under weak or no flow in rescaled variables, based on the solidification rate rather than the external flow speed, which is convenient for displaying results involving weak or no flow. The comparison uses the results of [16] and [20]. The value of $\Gamma_{s}$, in rescaled variables, is lower for $\mathcal{V} \gg 1$ than for $\mathcal{V}=0$ or $\mathcal{V} \ll 1$. That is, the range of surface energies for which instabilities occur is smaller for $\mathcal{V} \gg 1$, indicating that strong flow is stabilizing.

Fig. 6 also shows that the critical wavenumber decreases with $\Gamma$ as $\Gamma \rightarrow \Gamma_{s}$ for each of the modes of instability and that the differences between small and large magnitudes of the external flow become apparent only for large surface energies.

Both the critical wavenumber and the cutoff wavenumber decrease with $\mathcal{V}$, as shown in Fig. 7. In contrast to results for no flow and weak flow, under strong flow there is a nonzero small-wavenumber cutoff $\alpha_{o s}$, such that there are no instabilities for $\alpha<\alpha_{o s}$. Long waves are no longer preferred. 


\section{$5 \quad$ Physical Scalings}

In many physical and experimental scenarios, it is natural to adopt scalings that allow one to specify a measure of the solute concentration and solidification speed as externally controllable parameters. This can by done if capillary scales $l \sim\left(\gamma T_{M} /\left(L_{\nu} G\right)\right)^{1 / 2}, t \sim \gamma T_{M} /\left(L_{\nu} G D_{l}\right)$ are chosen to scale length and time as in [20], rather than the diffusion scales that we have used, which are convenient for the analysis.

We can rescale length and time against capillary scales by adopting the following transformation:

$$
\begin{aligned}
& \mathcal{M}=\mathscr{V} \mathscr{C} \mathcal{V}, \quad \Gamma=\mathscr{V} \mathscr{C}^{-1} \mathcal{V}, \quad \beta=\mathscr{B} \mathscr{V} \mathcal{V}, \quad(0 a-c) \\
& \mathcal{U}=\mathscr{V} \mathscr{N}_{\mathscr{C}^{-1}} \mathcal{V}, \quad \boldsymbol{k}=\mathscr{A}^{-1} \mathcal{V}, \quad \sigma=\mathscr{S}^{-2} \mathcal{V}^{2} . \quad(1 a-c)
\end{aligned}
$$

This is a rescaled version of the transformation used in [20].

Neutral curves for the two modes of instability present under no flow (the cellular mode and the oscillatory mode), separating regions of stability from regions of instability, are shown in the rescaled variables in $(\mathscr{C}, \mathscr{V})$ space in Fig. 8. Overlain in this figure is also the neutral curve resulting from a strong and weak imposed shear flow. The region of instability is much smaller under strong flow. Specifically, instabilities are seen only for smaller solidification rates. The upper bound for the solidification rate, above which no instabilities occur, is an order of magnitude lower for $\mathscr{V} \gg 1$ than under no flow. This implies that introducing a strong external shear flow is an effective way to reduce instabilities at large solidification rates. The lower bound, under which no instabilities occur, remains mainly unaffected.

Such stabilization via a strong external flow is similar to the stabilization of instabilities of thin liquid films via a shear flow, which produces an interfacial viscous-capillary wave capable of postponing and even eliminating instabilities completely [15]. The latter stabilization occurs in a nonlinear regime, in contrast to the mechanisms explored in this paper.

The wavenumbers, for which these instabilities occur under strong flow come close to the largest wavenumbers seen for results under no flow, and are bounded away from zero in contrast to results under no flow, as seen in Fig. 9. Fig. 9 shows a cross section of the neutral curves displayed in Fig. 8 at a fixed concentration $\mathscr{C}$. The two modes of instability under no flow become replaced by a single, oscillatory mode for $\mathcal{V} \gg 1$ of larger wavelengths and smaller solidification rates.

\section{Conclusions}

We have examined the effects of an imposed shear flow on the directional solidification of a binary alloy. The shear flow is imposed parallel to the solidification front and is of magnitude much larger than that of the 
solidification rate. The solid-liquid interface is allowed to depart from thermodynamic equilibrium.

Without any flow, the solid-liquid interface is susceptible to the onset of two modes of instability: a steady, cellular mode and a time-periodic, pulsatile mode $(\alpha=0)$ as seen in [20]. Once a weak flow is imposed, travelling waves appear and the first of these modes becomes weakly oscillatory, as shown in [16]. Even though there is no longer a sharp transition between these, the modes are nevertheless distininguishable.

This is no longer the case once the magnitude of the flow is large, as the two modes of instability, seen under no flow and weak flow, merge into a single mode. This mode is oscillatory $(\alpha \neq 0, \omega \neq 0)$ and occurs for wavenumbers that are close to the largest wavenumbers seen without flow, see Fig. 3 . In contrast to the scenario for the oscillatory mode under no flow, the critical wavenumber remains nonzero, despite being small. This means that the preferred mode induces spatial structure under strong flow in contrast to the spatially homogenous, oscillatory modes preferred by the system under no flow.

The length scale of the cellular structure typical of rapidly solidified materials under which the effect of flow is negligible has been additionally found to scale consistently with the cooling rate [see, e.g., 26]. Under strong flow, such structure no longer occurs and is replaced by modes that are oscillatory in the solidification direction. With strong flow, the dependence of the scale of these on the cooling rate may be controlled by the enhanced heat transport resulting from convection within the melt pool.

The critical $\mathcal{M}^{-1}$, as defined by the neutral curve, decreases with $\mathcal{V}$. The stronger the flow, the smaller the region of instability in parameter space. The critical and cutoff wavenumbers also both decrease with $\mathcal{V}$, meaning that instabilities under strong flow favor small wavenumbers.

Just as under no flow or weak flow, surface energy is seen to stabilize the system up to an absolute stability boundary $\Gamma=\Gamma_{s}$, which, under an appropriate rescaling based on the solidification rate rather than the external flow rate, is smaller under large flow rates than under small or no flow. Surface energies higher than this completely stabilize the interface. Instabilities under strong flow are seen for a smaller range of surface energies. A difference is that long waves are preferred near the absolute stability limit under no flow, but the system selects a distinct, critical wavelength near absolute stability once the magnitude of the flow is large. The critical wavenumber, as well as the cutoff wavenumber, both decrease towards this with the surface energy as it approaches the absolute stability limit.

A distinguishing feature, perhaps of interest for industrial applications, is that lower solidification rates are necessary for any instability to occur under strong flow than with no flow at all, or with weak flow. Inducing a strong shear flow is therefore an effective way to eliminate interfacial instabilities at high solidification rates. Such stabilization is similar to that of instabilities of thin liquid films, which may be postponed or even eliminated when a shear flow is introduced [15]. However, flow does not make a significant difference to stability thresholds 
at low solidification rates.

The triangular overlap region between the two primary modes of instability under no flow vanishes at large flow rates, which suggests that banding will not occur at large external-flow velocities. This loss of banding requires experimental verification. All experimental data on banding occur within this overlap region [20], but there have been no experiments conducted on instabilities under rapid solidification with flow. Introducing a large external flow may be a way of influencing the microstructure during rapid solidification, as a means to eliminate banding.

\section{Acknowledgements}

This work is supported by the National Institute of Standards and Technology [grant number 70NANB14H012] as part of the Center for Hierarchical Material Design (CHiMaD).

\section{References}

[1] M. J. Aziz. Model for solute redistribution during rapid solidification. J. Appl. Phys., 53(2):1158-1168, 1982.

[2] J. C. Baker and J. W. Cahn. In Solidification, page 23. Am. Soc. Metals, Metals Park, Ohio, 1971.

[3] W. J. Boettinger and S. R. Coriell. Microstructure formation in rapidly solidified alloys. In P. R. Sahm, H. Jones, and C. M. Adam, editors, Rapid Solidification Materials and Technologies. NATO ASI Series, Series E: Applied Sciences, 1986.

[4] W. J. Boettinger and J. H. Perepezko. Fundamentals of rapid solidification. In S. K. Das, B. H. Kear, and C. M. Adam, editors, Rapid Solidified Crystalline Alloys. Proceedings of a TMS-AIME Northeast Regional Meeting, Metallurgical Soc of AIME, 1985.

[5] S. Y. Chin, Y. C. Poh, A-C Kohler, J. T. Compton, L. L. Hsu., K. M. Lau., S. Kim, B. W. Lee., F. Y. Lee, and S. K. Sia. Additive manufacturing of hydrogel-based materials for next-generation implantable medical devices. Science Robotics, 2(2), 2017.

[6] S. R. Coriell and G. B. McFadden. Morphological stability. In D. T. J. Hurle, editor, Handbook of Crystal Growth, volume 1, pages 785-858. Elsevier, Amsterdam, 1993.

[7] S. H. Davis. Theory of Solidification. Cambridge University Press, 2001. 
[8] S. A. Forth and A. A. Wheeler. Hydrodynamic and morphological stability of the unidirectional solidification of a freezing binary alloy: a simple model. J. Fluid Mech., 202:339-366, 1989.

[9] I. Gibson, D. Rosen, and B. Stucker. Additive Manufacturing Technologies: 3D Printing, Rapid Prototyping and Direct Digital Manufacturing. Springer, New York,, 2015.

[10] Y. He, G. Xue, and J. Fu. Fabrication of low cost soft tissue prostheses with the desktop 3D printer. Sci. Rep., 4, 2014. 6973.

[11] A.K. Hobbs and Ph. Metzener. Long-wave instabilities in directional solidification with remote flow. J. Cryst. Growth, 112:539-553, 1991.

[12] D. C. Hofmann, S. Roberts, R. Otis, J. Kolodziejska, R. P. Dillon, J-O Suh, A. A. Shapiro, Z-K Liu, and J-P Borgonia. Developing gradient metal alloys through radial deposition additive manufacturing. Sci. Rep., 4, 2014. 5357.

[13] K.A. Jackson, G.H. Gilmer, and H.J. Leamy. Solute trapping. In C.W. White and P.S. Peercy, editors, Laser and Electron Beam Processing of Materials, pages 104-110. Academic Press, 1980.

[14] S. A. Khairallah, A. T. Anderson, A. Rubenchik, and W. E. King. Laser powder-bed fusion additive manufacturing: Physics of complex melt flow and formation mechanisms of pores, spatter, and denudation zones. Acta Materialia., 108:36-45, 2016.

[15] E. Kirkinis and S. H. Davis. Stabilization mechanisms in the evolution of thin liquid-films. Proc. R. Soc. A, 471(20150651), 2015.

[16] K. N. Kowal, S. H. Davis, and P. W. Voorhees. Instabilities in rapid directional solidification under weak flow. Phys. Rev. E, 96:062802, 2017.

[17] K. N. Kowal, S. H. Davis, and P. W. Voorhees. Thermocapillary instabilities in a horizontal liquid layer under partial basal slip. J. Fluid Mech., 2018. Submitted.

[18] W. Kurz and D. J. Fisher. Fundamentals of Solidification. Trans. Tech. Publ., 1989.

[19] M. J. Matthews, G. Guss, S. A. Khairallah, A. M. Rubenchik, P. J. Depond, and W. E. King. Denudation of metal powder layers in laser powder bed fusion processes. Acta Materialia, 114:33-42, 2016.

[20] G. J. Merchant and S. H. Davis. Morphological instability in rapid directional solidification. Acta metall, mater., 38(12):2683-2693, 1990. 
[21] J. S. Miller, K. R. Stevens, M. T. Yang, B. M. Baker, D-H T. Nguyen, D M. Cohen, E. Toro, A. A. Chen, P. A. Galie, X. Yu, R. Chaturvedi, S. N. Bhatia, and C. S. Chen. Rapid casting of patterned vascular networks for perfusable engineered three-dimensional tissues. Nature Mater., 11:768-774, 2012.

[22] T. Mukherjee, J. S. Zuback, A. De, and T. DebRoy. Printability of alloys for additive manufacturing. Sci. Rep., 6, 2016. 19717.

[23] W. W. Mullins and R. F. Sekerka. Stability of a planar interface during solidification of a dilute binary alloy. J. Appl. Phys., 35(2):444-451, 1964.

[24] S. V. Murphy and A. Atala. 3D bioprinting of tissues and organs. Nature Biotechnol., 8:773-785, 2014.

[25] W. J. Sames, F. A. List, S. Pannala, R. R. Dehoff, and S. S. Babu. The metallurgy and processing science of metal additive manufacturing. International Materials Reviews, 61(5):315-360, 2016.

[26] M. Tang, P. C. Pistorius, S. Narra, and J. L. Beuth. Rapid solidification: Selective laser melting of AlSi10Mg. JOM, 68(3):960-966, 2016.

[27] U. G. K. Wegst., H. Bai, E. Saiz, A. P. Tomsia, and R. O. Ritchie. Bioinspired structural materials. Nature Materials., 14(1):23-36, 2015.

[28] W. K. C. Yung, B. Sun, Z. Meng, J. Huang, Y. Jin, H. S. Choy, Z. Cai, G. Li, C. L. Ho, J. Yang, and W. Y. Wong. Additive and photochemical manufacturing of copper. Sci. Rep., 6, 2016. 39584.

[29] Y. Zheng, Z. He, Y. Gao, and J. Liu. Direct desktop printed-circuits-on-paper flexible electronics. Sci. Rep., 3, 2013. 1786 . 